\title{
Computational complexity for the two-point block method
}

\begin{abstract}
In this paper, we discussed and compared the computational complexity for two-point block method and one-point method of Adams type. The computational complexity for both methods is determined based on the number of arithmetic operations performed and expressed in $\mathrm{O}(\mathrm{n})$. These two methods will be used to solve two-point second order boundary value problem directly and implemented using variable step size strategy adapted with the multiple shooting technique via three-step iterative method. Two numerical examples will be tested. The results show that the computational complexity of these methods is reliable to estimate the cost of these methods in term of the execution time. We conclude that the twopoint block method has better computational performance compare to the one-point method as the total number of steps is larger.
\end{abstract}

Keyword: Block method; Boundary value problem; Computational complexity 